\title{
Multi-cancer computational analysis reveals invasion-associated variant of desmoplastic reaction involving INHBA, THBS2 and COL11A1
}

\author{
Hoon Kim', John Watkinson ${ }^{1}$, Vinay Varadan², Dimitris Anastassiou ${ }^{1 *}$
}

\begin{abstract}
Background: Despite extensive research, the details of the biological mechanisms by which cancer cells acquire motility and invasiveness are largely unknown. This study identifies an invasion associated gene signature shedding light on these mechanisms.

Methods: We analyze data from multiple cancers using a novel computational method identifying sets of genes whose coordinated overexpression indicates the presence of a particular phenotype, in this case high-stage cancer.

Results: We conclude that there is one shared "core" metastasis-associated gene expression signature corresponding to a specific variant of stromal desmoplastic reaction, present in a large subset of samples that have exceeded a threshold of invasive transition specific to each cancer, indicating that the corresponding biological mechanism is triggered at that point. For example this threshold is reached at stage IIIc in ovarian cancer and at stage II in colorectal cancer. Therefore, its presence indicates that the corresponding stage has been reached. It has several features, such as coordinated overexpression of particular collagens, mainly COL11A1 and other genes, mainly THBS2 and INHBA. The composition of the overexpressed genes indicates invasion-facilitating altered proteolysis in the extracellular matrix. The prominent presence in the signature of INHBA in all cancers strongly suggests a biological mechanism centered on activin A induced TGF- $\beta$ signaling, because activin A is a member of the TGF- $\beta$ superfamily consisting of an INHBA homodimer. Furthermore, we establish that the signature is predictive of neoadjuvant therapy response in at least one breast cancer data set.

Conclusions: Therefore, these results can be used for developing high specificity biomarkers sensing cancer invasion and predicting response to neoadjuvant therapy, as well as potential multi-cancer metastasis inhibiting therapeutics targeting the corresponding biological mechanism.
\end{abstract}

\section{Background}

There is currently great interest in identifying the biological mechanisms for the acquisition of motility and invasiveness in cancer. It has been hypothesized [1,2] that they often involve some form of epithelial-mesenchymal transition (EMT), significant involvement of the tumor microenvironment $[3,4]$ and the presence of activated fibroblasts in the "reactive" desmoplastic stroma of tumors, referred to as "cancer associated fibroblasts" (CAFs) [5,6].

\footnotetext{
* Correspondence: anastas@ee.columbia.edu

${ }^{1}$ Center for Computational Biology and Bioinformatics and Department of Electrical Engineering, Columbia University, New York, NY, USA

Full list of author information is available at the end of the article
}

A study [7] of serous papillary ovarian carcinomas, comparing the gene expression profiles of primary vs. omental metastatic tumors, identified 156 differentially expressed genes. To investigate the significance of these genes in an independent rich data set we performed hierarchical clustering, using only these genes, on The Cancer Genome Atlas (TCGA) gene expression data set consisting of 377 ovarian cancer samples containing staging information. The resulting heat map revealed a prominent block of about 100 highly overexpressed genes in 94 samples (Additional file 1). Remarkably, we found that none of the 41 samples from tumors of stages IIIb and below were among the 94 samples. This cannot be due to chance $\left(\mathrm{p}=4 \times 10^{-6}\right)$, leading to the hypothesis that coordinated overexpression of these 
genes implies that the tumor has progressed to at least stage IIIc.

To further validate this hypothesis and test if similar versions apply to other cancers, we developed a computational technique identifying in an unbiased manner coordinatedly overexpressed genes associated with a phenotype (such as transition to a particular stage). Our results consistently rediscover the same "core" signature of overexpressed genes, confirming the hypothesis. We found that the signature is present in multiple cancers, each of which has its own features involving additional genes, but the core signature is common.

As evidenced by the presence of $F A P$ (fibroblast activation protein) and ACTA2 (actin, alpha 2, also known as $\alpha-S M A$, alpha-smooth muscle actin) in the set of overexpressed genes, the signature suggests a variant of stromal desmoplastic reaction. As further evidence, it is known ([6], p. 546) that activated fibroblasts (myofibroblasts) construct the desmoplastic stroma through the secretion of large amounts of collagen, fibronectin (FN1) and proteoglycans, and they secrete various proteases such as urokinase plasminogen activator (PLAU) and matrix metalloproteinases (MMPs). This list precisely describes most of the genes appearing in the signature, and its precise composition (e.g., having COL11A1 as the prominent collagen, MMP11 as the prominent metalloproteinase, and $\mathrm{CDH} 11$ as the prominent cadherin) points to a particular variant of CAFs, to which we refer as "metastasis associated fibroblasts" (MAFs). Indeed, the resulting proteolytic remodeling at the invasive edge of the tumor is thought to facilitate the initial invasive stage of the metastatic process by "excavating passageways" ([6], p. 621) through the extracellular matrix for the cancer cells to go through. Accordingly, in the following we refer to the corresponding gene expression signature and biological mechanism as "the MAF signature" and "the MAF mechanism," respectively.

\section{Methods}

\section{List of data sets}

The list of data sets in the paper is given in Table 1 . They were identified by searching for rich data sets focused on a specific cancer in two public databases, The Cancer Genome Atlas and the Gene Expression Omnibus data depository. Furthermore, for the data sets initially used to infer the gene signature we required that they have well annotated staging information associated with the samples and that they contain a significant number of samples in both lower and higher stages so that we could compare the expression profiles across stages.

\section{Extreme Value Association (EVA)}

Since we aim to discover a set of genes that are coordinately overexpressed only in a subset of the "high stage" samples, we developed a special measure of association between the gene and the binary ("high stage" vs. "low stage") phenotype that naturally fits this description, ignoring the expression levels of the genes outside the region of overexpression, which we call "extreme value association" (EVA). The same measure can identify coordinately silenced genes, but we did not find any such genes across various cancer types.

The EVA metric is the minimum p-value of biased partitions over all subsets of samples with highest expression values of the gene. In other words, suppose that there are totally $M$ samples, out of which $N$ are "low stage" and $M-N$ are "high stage," and we select the $m$ samples with the highest gene expression values, out of which $n$ are "low stage" and $m-n$ are "high stage." Under the assumption that gene expression values are uncorrelated with the phenotype, the probability that there will be at most $n$ "low stage" samples among the selected $m$ samples is given by the cumulative hypergeometric probability $h(x \leq n ; M, N, m)$. The EVA metric is then defined as equal to $-\log _{10}$ of the minimum of these probabilities over all possible choices of $m$, in which $m$ ranges from 1 to $M$ (note that $n$ depends on $m$ ). For example, assume that there are 250 high-stage samples and 50 low-stage samples for a total of 300 samples. Furthermore, assume that the 100 samples with the highest values of a particular gene contain 99 high-stage samples and one low stage sample. In that case, $h(x \leq 1 ; 300,50,100)$ can be evaluated using the MATLAB function hypercdf $(1,300,50,100)=5 \times 10^{-9}$, resulting in the EVA metric for that gene of at least $-\log _{10}\left(5 \times 10^{-9}\right)=8.3$. If the $101^{\text {th }}$ sample is also highstage, then the EVA metric of the gene will be even higher. Note that, once the highest value is reached, the sorting arrangement of the remaining samples is irrelevant, reflecting the hypothesis that only the extreme values are associated with the phenotype. Figure 1 shows the values of the cumulative hypergeometric probability for the COL11A1 gene using the TCGA ovarian cancer data set and the staging threshold for the definition of the binary phenotype set between IIIb and IIIc. The maximum (8.31) occurs when $m=133$.

We then developed a mechanistic and unbiased (only dependent on the phenotype) algorithm, which, when given a gene expression data set for a number of samples labeled "high stage" or "low stage," leads to a selection of genes that are coordinatedly overexpressed only in high-stage samples, ignoring the effect of the rest, thus precisely reflecting the observed phenomenon. We first select the top 100 genes that rank highest according to the EVA metric criterion using a mixture analysis (selecting the minimum p-value) of both overexpressed and silenced genes. Using this set of genes only, we perform k-means clustering with gap statistic [8]. At that 
Table 1 Lists of Data sets in the paper

\begin{tabular}{|c|c|c|c|c|}
\hline Data set name & Source Site & GEO Accession & Affymetrix platform & Sample size \\
\hline TCGA Ovarian Cancer & The Cancer Genome Atlas & & HT_HG-U133A ${ }^{a}$ & 377 \\
\hline CCR Ovarian Cancer & Gene Expression Omnibus & GSE9891 & HG-U133_Plus_2 ${ }^{\mathbf{b}}$ & 285 \\
\hline CCR Colon Cancer & Gene Expression Omnibus & GSE14333 & HG-U133_Plus_2 & 290 \\
\hline Moffitt Colon Cancer & Gene Expression Omnibus & GSE17536 & HG-U133_Plus_2 & 177 \\
\hline Singapore Gastric Cancer & Gene Expression Omnibus & GSE15459 & HG-U133_Plus_2 & 200 \\
\hline CCR Breast Cancer & Gene Expression Omnibus & GSE7390 & HG-U133A $A^{c}$ & 198 \\
\hline Wang Breast Cancer & Gene Expression Omnibus & GSE2034 & HG-U133A & 286 \\
\hline Samsung Lung Cancer & Gene Expression Omnibus & GSE8894 & HG-U133_Plus_2 & 138 \\
\hline Bild Lung Cancer & Gene Expression Omnibus & GSE3141 & HG-U133_Plus_2 & 111 \\
\hline Neuroblastoma tumor & Gene Expression Omnibus & GSE3960 & HG_U95Av2 & 102 \\
\hline Neoadjuvant Breast Cancer & Gene Expression Omnibus & GSE4779 & U133_X3P & {$[65] 102^{e}$} \\
\hline Postmortem - normal & Gene Expression Omnibus & GSE3526 & HG-U133_Plus_2 & 353 \\
\hline Human body index - normal & Gene Expression Omnibus & GSE7307 & HG-U133_Plus_2 & $504[677]^{f}$ \\
\hline
\end{tabular}

${ }^{a}$ Affymetrix HT Human Genome U133A Array.

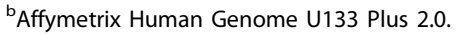

'Affymetrix Human Genome U133A.

${ }^{d} 270$ out of 341 samples are tumor samples.

${ }^{\mathrm{e}} 65$ out of 102 are ER-negative samples.

$f_{504}$ out of 677 samples are normal samples.

step, if indeed the genes are coordinately overexpressed, they will align well in the heat map. This leads to the selection of the samples belonging to the cluster most associated with the high/low stage phenotype - call this the set of "EVA-based samples." Next, we define a "clean" MAF phenotype, contrasting the samples that are: (a) both "EVA based" and "high-stage" against (b) the samples that are both "non EVA-based" and "low stage." If the number of samples is sufficiently large, this "clean" phenotype provides the sharpest way by which we can identify the genes that are most associated with the observed phenomenon of metastasis-associated coordinated overexpression. We then rank the genes and compute their multiple-test-corrected p-values

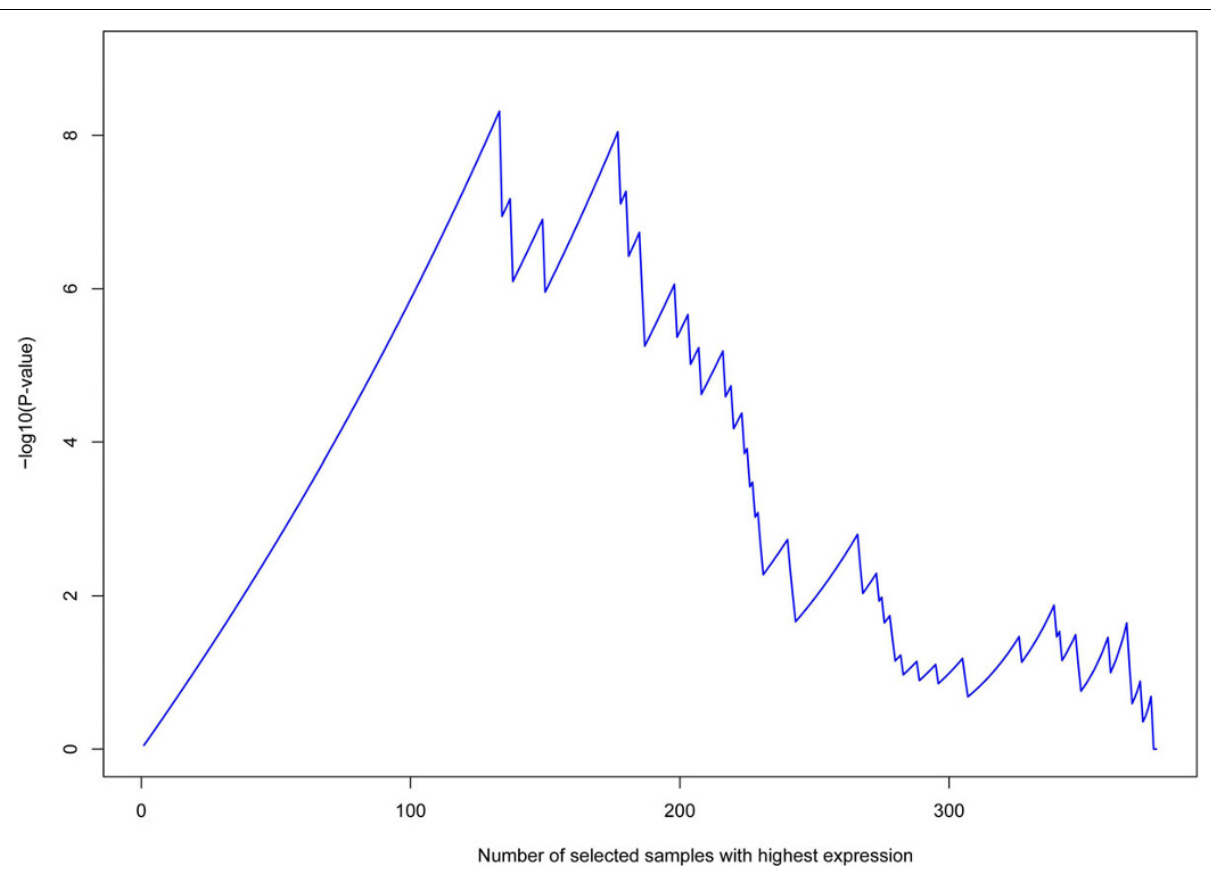

Figure 1 Evaluation of the EVA metric for gene COL11A1 in the TCGA ovarian cancer data set. 
using a Wilcoxon rank-sum test using the "clean" phenotype and select the genes for which $\mathrm{p}<10^{-3}$ after Bonferroni correction. Finally, we find the intersection of these selected gene sets over all cancer expression data sets and rank them in terms of fold change.

For a data set with $n$ samples and $m$ genes, the EVA algorithm computes cumulative hypergeometric distribution probabilities $\mathrm{nm}$ times. This can be computationally intensive, so we devised a low-complexity implementation algorithm described in Additional file 2.

\section{Mutual Information and Synergy}

Assuming that two variables, such as the expression levels of two genes $G_{1}$ and $G_{2}$, are governed by a joint probability density $p_{12}$ with corresponding marginals $p_{1}$ and $p_{2}$ and using simplified notation, the mutual information $I\left(G_{1} ; G_{2}\right)$ is a general measure of correlation and is defined as the expected value $E\left\{\log \frac{p_{12}}{p_{1} p_{2}}\right\}$. The synergy of two variables $G_{1}, G_{2}$, with respect to a third variable $G_{3}$ is [9] equal to $I\left(G_{1}, G_{2} ; G_{3}\right)-\left[I\left(G_{1} ; G_{3}\right)+\right.$ $\left.I\left(G_{2} ; G_{3}\right)\right]$, i.e., the part of the association of the pair $G_{1}$, $G_{2}$ with $G_{3}$ that is purely due to a synergistic cooperation between $G_{1}$ and $G_{2}$ (the "whole" minus the sum of the "parts"). We used a $3^{\text {rd }}$ order B-spline-based mutual information estimator dividing the observation space into six equally spaced bins in each dimension.

\section{P-value estimation for mutual information and synergy}

We applied a permutation-based approach accounting for multiple test correction: We did 100 permutation experiments of the class labels, saving the corresponding 100 highest values after doing exhaustive search in each permutation experiment. Using the set of these 100 highest value scores, we obtained the maximum likelihood estimates of the location parameter and the scale parameter of the Gumbel (type-I extreme value) distribution, resulting in a cumulative density function $F$. The p-value of an actual score $x_{0}$ is then $1-F\left(x_{0}\right)$ under the null hypothesis of no association with phenotype. Similarly, for the synergistic pair, we found the top-scoring synergy in 100 data sets that were identical to the original except that the phenotype values were randomly permuted on each, and the top permuted synergy scores were modeled, as above, with the Gumbel distribution.

\section{Results}

\section{Identification of MAF signature genes from staging} information in four data sets

We performed the EVA algorithm on four rich gene expression data sets, two from ovarian cancer, the one from TCGA and another one [10], and two from colorectal cancer $[11,12]$ accompanied by staging information. We performed the algorithm multiple times using the different possible cutoff thresholds defining the phenotype, finding that, in all cases, it is defined as exceeding stage IIIb in each of the ovarian data sets and stage I in each of the colorectal data sets. Interestingly, several among the "metastasis-associated genes" identified in [7] as present in omental metastasis of ovarian cancer were also identified in [10] as belonging to a subtype of ovarian cancer characterized by extensive desmoplasia, which contains the MAF signature.

Remarkably, we found that there were 137 genes (listed in Additional file 3), each of which had Bonferroni-corrected $\mathrm{p}<10^{-3}$ in all four data sets. Table 2 shows a list of these genes with average log fold change greater than 2. The top ranked gene was COL11A1 (probe 37892_at). Again, these genes were found purely as a result of their association with the staging phenotype in all four cancers. Gene Ontology enrichment testing of these genes identified cell adhesion, extracellular matrix and collagen fibril organization. It turns out that use of other standard correlation measures instead of the EVA measure in the same algorithms leads to the same results, because the overall correlation of these genes with the phenotype is strong merely as a result of the genes' overexpression in some high-stage samples alone. The EVA method has the additional advantage of providing an estimate of the size of that subset of high-stage samples.

We then did an extensive literature search aimed at identifying other studies in which at least some of these genes were identified as differentially expressed in various stages of other cancers. We even scrutinized studies in which none of the genes were mentioned in the main text, by looking at their supplementary data and reranking particular columns of genes in terms of their fold changes, from genes containing numerous genes. Although most of our results were negative, we were able to produce cancer gene lists with striking similarity (Table 3) to our own list (Table 2) in three studies of breast [13], gastric [14] and pancreatic [15] cancer.

Specifically, a breast cancer study [13] comparing ductal carcinomas in situ (DCIS) with invasive ductal carcinoma (IDC) had a list of genes upregulated in IDC that had similarities to those we had identified, and the topranked gene was again COL11A1 (probe 37892_at) with $\log$ fold change of 6.50 , while the next highest (4.08) corresponded to another probe of COL11A1, followed by a probe of COL1OA1. Second, a study [14] comparing early gastric cancer (EGC) with advanced gastric cancer (AGC) - roughly separating stages I and II - also identified a similar differentially expressed gene list of which again COL11A1 (probe 37892_at) was at the top (log fold change: 4.26) followed by COL10A1 and FAP. Third, a study of pancreatic ductal adenocarcinoma [15] 
Table 2 Top-ranked genes associated with carcinoma stage in four ovarian and colorectal cancers

\begin{tabular}{|c|c|c|}
\hline Probe Set $^{\mathrm{a}}$ & Gene & Log FC \\
\hline 37892_at & COL11A1 & 3.94 \\
\hline 217428_s_at & COLIOA1 & 3.55 \\
\hline 204320_at & COL11A1 & 3.39 \\
\hline 210809_s_at & POSTN & 3.14 \\
\hline 206439_at & EPYC & 3.12 \\
\hline 219087_at & ASPN & 2.99 \\
\hline 205941_s_at & COLIOA1 & 2.88 \\
\hline 203083_at & THBS2 & 2.81 \\
\hline 209955_s_at & FAP & 2.73 \\
\hline 215446_s_at & LOX & 2.63 \\
\hline 204051_s_at & SFRP4 & 2.53 \\
\hline 210511_s_at & INHBA & 2.52 \\
\hline 215646_s_at & VCAN & 2.50 \\
\hline 218469_at & GREM1 & 2.48 \\
\hline 209758_s_at & MFAP5 & 2.42 \\
\hline 218468_s_at & GREM1 & 2.35 \\
\hline 212353_at & SULF1 & 2.34 \\
\hline 221730_at & COL5A2 & 2.34 \\
\hline 211571_s_at & VCAN & 2.33 \\
\hline 204619_s_at & VCAN & 2.33 \\
\hline 205713_s_at & COMP & 2.31 \\
\hline 221731_x_at & VCAN & 2.27 \\
\hline 204620_s_at & VCAN & 2.26 \\
\hline 201150_s_at & TIMP3 & 2.25 \\
\hline 221729_at & COL5A2 & 2.24 \\
\hline 212354_at & SULF1 & 2.23 \\
\hline 212489_at & COL5A1 & 2.22 \\
\hline 213790_at & ADAM12 & 2.21 \\
\hline 212488_at & COL5A1 & 2.20 \\
\hline 201147_s_at & TIMP3 & 2.19 \\
\hline 204457_s_at & GAS1 & 2.17 \\
\hline 206026_s_at & TNFAIP6 & 2.14 \\
\hline 202952_s_at & ADAM12 & 2.12 \\
\hline 202766_s_at & FBN1 & 2.08 \\
\hline 212344_at & SULF1 & 2.07 \\
\hline 202311_s_at & COLIA1 & 2.05 \\
\hline 209335_at & $D C N$ & 2.01 \\
\hline
\end{tabular}

${ }^{a}$ Affymetrix probe sets.

All genes have Bonferroni corrected $p<10^{-3}$ in each of the four data sets.

identified a list of genes overexpressed in whole tumor tissue versus normal pancreatic tissue, in which COL11A1 (probe 37892_at) is again prominent and the top entry (log fold change 5.15) was INHBA, supportive of our hypothesis of activin A induced TGF- $\beta$ signaling. The presence of the MAF signature in the latter study indicates that pancreatic cancer had already become invasive in most cases before the biopsy. The prominent desmoplastic reaction in pancreatic cancers (which contains the MAF signature) has recently been increasingly recognized as a "foe" [16] that could lead to new therapeutic strategies targeting stromal cells to inhibit cancer. Finally, we realized that COL11A1 has been identified as a potential metastasis-associated gene in other types of cancer as well, such as in lung [17], and oral cavity [18], suggesting that the MAF signature may be present in a subset of high stage samples of most if not all epithelial cancers.

\section{Using COL11A1 as proxy for the MAF signature in the absence of staging information}

In those cases as well as in our own findings, there was prominent presence of COL11A1 (probe 37892_at). This remarkable consistent strong association of COL11A1 with the staging phenotype (specific to each cancer type) suggests that it could be used as a "proxy" of the MAF signature. This would allow us to improve on the gene list of Table 2 by making use of numerous publicly available gene expression data sets of cancers of many types, even without any staging information, as long as the MAF signature is present in a sizeable subset of them, aiming at finding the "intersection" of the associated factors in these sets, revealing the "core" of the MAF biological mechanism.

As a first step for this task, we identified the genes that are consistently highest associated with COL11A1. Additional file 4 shows a listing of genes in nine cancer data sets, while Table 4 shows an aggregate list of the top 100 genes ranked in terms of their association (mutual information) with COL11A1. The list is similar to the phenotype-based gene ranking (Table 2). In addition to a few collagens of type XI, X, V, and I, the top ranked genes are thrombospondin-2 (THBS2), inhibin beta A (INHBA), leucine rich repeat containing 15 (LRRC15), versican $(V C A N)$, fibroblast activation protein $(F A P)$, and matrix metallopeptidase 11 (MMP11) aka stromelysin 3 . The presence of $F A P$ indicates a general desmoplastic reaction and is not, by itself, sufficient for inferring the MAF signature.

Furthermore, contrary to all other genes, COL11A1 was uniquely not associated with any of these genes in noncancerous samples, further supporting the hypothesis that it can be used as a proxy for the MAF signature. Our results indicate that THBS2 and INHBA, top ranked in Table 4 except for collagens, are the most important players in the MAF mechanism. Figure 2 demonstrates this striking coexpression in data sets of cancer samples, but not in noncancerous samples, in the form of scatter plots. We have consistently validated this behavior in the cancerous and noncancerous data sets we tested.

As a second step, we identified gene pairs that are highest associated with COL11A1 jointly, but not individually, and therefore they would not appear in the previous list. For this task we ranked gene pairs according to their synergy [9] with $C O L 11 A 1$, using the 
Table 3 Gene lists produced from information provided in the corresponding papers for other cancers

\begin{tabular}{|c|c|c|c|c|c|c|c|c|}
\hline \multicolumn{3}{|c|}{ 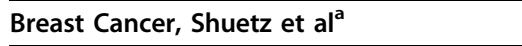 } & \multicolumn{3}{|c|}{ Gastric cancer, Vecchi et al ${ }^{b}$} & \multicolumn{3}{|c|}{ Pancreatic cancer, Badea et al ${ }^{c}$} \\
\hline Probe Set $^{d}$ & Gene Symbol & Log FC & Probe Set $^{d}$ & Gene Symbol & Log FC & Probe Set $^{d}$ & Gene Symbol & Log FC \\
\hline 37892_at & COL11A1 & 6.50 & 37892_at & COL11A1 & 4.26 & 227140_at & INHBA & 5.15 \\
\hline 204320_at & COL11A1 & 4.08 & 217428_s_at & COL10A1 & 4.15 & 217428_s_at & COL10A1 & 5.00 \\
\hline 217428_s_at & COLIOA1 & 4.07 & 209955_s_at & FAP & 3.40 & 1555778_a_at & POSTN & 4.92 \\
\hline 213764_s_at & MFAP5 & 3.73 & 235458_at & HAVCR2 & 3.30 & 212353_at & SULF1 & 4.63 \\
\hline 213909_at & LRRC15 & 3.61 & 204320_at & COL11A1 & 3.28 & 226237_at & COL8A1 & 4.60 \\
\hline 205941_s_at & COLIOAI & 3.52 & 205941_s_at & COL10A1 & 3.21 & 37892_at & COL11A1 & 4.40 \\
\hline 210511_s_at & INHBA & 3.44 & 204052_s_at & SFRP4 & 2.90 & 225681_at & CTHRC1 & 4.38 \\
\hline 202766_s_at & FBN1 & 3.43 & 226930_at & FNDC1 & 2.85 & 202311_s_at & COLIA1 & 4.12 \\
\hline 212353_at & SULF1 & 3.35 & 227140_at & INHBA & 2.77 & 203083_at & THBS2 & 3.97 \\
\hline 218468_s_at & GREM1 & 3.35 & 209875_s_at & SPP1 & 2.77 & 227566_at & HNT & 3.90 \\
\hline 215446_s_at & LOX & 3.22 & 205422_s_at & ITGBLI & 2.63 & 204619_s_at & CSPG2 & 3.87 \\
\hline 221730_at & COL5A2 & 3.22 & 226311_at & - & 2.63 & 229802_at & WISP1 & 3.80 \\
\hline 218469_at & GREM1 & 3.20 & 222288_at & - & 2.62 & 212464_s_at & FN1 & 3.69 \\
\hline 212489_at & COL5A1 & 3.08 & 231993_at & - & 2.50 & 205713_s_at & COMP & 3.53 \\
\hline 203083_at & THBS2 & 2.99 & 226237_at & COL8A1 & 2.48 & 221729_at & COL5A2 & 3.38 \\
\hline 201505_at & LAMB1 & 2.97 & 223122_s_at & SFRP2 & 2.47 & 209955_s_at & $F A P$ & 3.37 \\
\hline 209955_s_at & FAP & 2.96 & 210511_s_at & INHBA & 2.43 & 229218_at & COL1A2 & 3.16 \\
\hline 209758_s_at & MFAP5 & 2.92 & 203819_s_at & IMP-3 & 2.39 & 209016_s_at & KRT7 & 3.13 \\
\hline 202363_at & SPOCK & 2.91 & 212464_s_at & FN1 & 2.36 & 210004_at & OLR1 & 3.03 \\
\hline 213241_at & $N Y-R E N-58$ & 2.90 & 212353_at & SULF1 & 2.35 & 219773_at & NOX4 & 3.02 \\
\hline 205479_s_at & PLAU & 2.89 & 227995_at & - & 2.34 & 218804_at & TMEM16A & 2.90 \\
\hline 206584_at & LY96 & 2.88 & 225681_at & CTHRCI & 2.30 & 238617_at & - & 2.87 \\
\hline 204475_at & MMP1 & 2.83 & 204457_s_at & GAS1 & 2.27 & 224694_at & ANTXR1 & 2.82 \\
\hline 202952_s_at & ADAM12 & 2.83 & 216442_x_at & FN1 & 2.25 & 228481_at & COX7A1 & 2.77 \\
\hline 201792_at & $A E B P 1$ & 2.81 & 223121_s_at & SFRP2 & 2.23 & 226311_at & ADAMTS2 & 2.76 \\
\hline 204114_at & NID2 & 2.81 & 211719_x_at & FN1 & 2.23 & 201792_at & $A E B P 1$ & 2.68 \\
\hline 213790_at & ADAM12 & 2.80 & 204776_at & THBS4 & 2.18 & 203021_at & SLPI & 2.65 \\
\hline 209156_s_at & COL6A2 & 2.77 & 210495_x_at & FN1 & 2.15 & 227314_at & ITGA2 & 2.58 \\
\hline 219179_at & DACT1 & 2.74 & 202800_at & SLCIA3 & 2.13 & 205499_at & SRPX2 & 2.44 \\
\hline 212488_at & COL5A1 & 2.73 & 214927_at & - & 2.11 & 226997_at & - & 2.41 \\
\hline 219087_at & $A S P N$ & 2.73 & 212354_at & SULF1 & 2.09 & 219179_at & DACT1 & 2.36 \\
\hline 204619_s_at & CSPG2 & 2.70 & 238654_at & LOC147645 & 2.06 & 203570_at & LOXL1 & 2.30 \\
\hline 204337_at & RGS4 & 2.69 & 213943_at & TWIST1 & 2.06 & 201850_at & CAPG & 2.25 \\
\hline 204620_s_at & CSPG2 & 2.69 & 236028_at & $I B S P$ & 2.05 & 222449_at & TMEPAI & 2.19 \\
\hline 212354_at & SULFI & 2.68 & 228481_at & POSTN & 2.00 & 227276_at & PLXDC2 & 2.16 \\
\hline
\end{tabular}

${ }^{a}$ Breast cancer list indicates genes overexpressed in invasive ductal carcinoma vs. ductal carcinoma in situ.

${ }^{\mathrm{b}}$ Gastric cancer list indicates genes overexpressed in early gastric cancer vs. advanced gastric cancer.

'Pancreatic cancer list indicates genes overexpressed in pancreatic ductal adenocarcinoma vs. normal pancreatic tissue.

${ }^{\mathrm{d}}$ Affymetrix probe sets.

computational method in [19], which could further facilitate biological discovery. For example, the scatter plots in Figure 3 show that genes ECM2 and TCF21 are jointly, but not individually, strongly associated with COL11A1 ( $\mathrm{p}<10^{-6}$, see Methods) in the two ovarian cancer data sets. Such findings are useful for developing biological hypotheses, e.g. in this particular case they suggest that in ovarian cancer the extracellular matrix protein 2 is associated with the MAF signature only when the TCF21 gene (a known mesenchymal-epithelial transition mediator) is downregulated.
The MAF signature exists even in non-epithelial cancers. Indeed, we confirmed that neuroblastoma also carries the MAF signature consistently associated with high stage: As shown in Additional file 5, none of 21 stage I samples have the signature $\left(\mathrm{p}=4 \times 10^{-4}\right)$, based on the genes highest associated with COL11A1.

\section{MicroRNAs and Methylated sites}

We only had miRNA and methylation data available for the TCGA ovarian data set. Using as measure the mutual information with COL11A1, we found many 
statistically significant miRNAs, among them hsa-miR22 and hsa-miR-152, as well as differentially methylated genes, such as SNAII and PRAME, suggesting a particularly complex biological mechanism (correlation with the MAF phenotype led to essentially the same lists with lower significance). Table 5 contains a list of the miRNAs, while Table 6 contains a list of the methylated genes (multiple test corrected $\mathrm{p}<10^{-16}$ in both cases, see Methods). SNAI1 (snail) methylation is particularly important as the gene is known as one of the most important EMT-related transcription factors. Instead, the strongest MAF-associated transcription factor is AEBP1. Many of the other EMT-related transcription factors, such as SNAI2, TWIST1, and ZEB1 are often overexpressed in the MAF signature, but SNAI1 is not (and, at least in ovarian carcinoma in which we have methylation data, this is due to its differentially methylated status). We believe that the lack of SNAI1 expression is a key distinguishing feature of the MAF signature, in which we observed neither SNAI1 overexpression nor CDH1 (E-cadherin) downregulation, at least on the mRNA level.

\section{Drug response}

Significantly, we also found that, at least in ER negative breast cancer, the MAF signature is associated with resistance to neoadjuvant FEC. This was demonstrated in [20] where a stromal "metagene" signature of 50 genes was defined based on $D C N$ (decorin). Although some of the MAF key genes (such as COL11A1 and THBS2) were not among these 50 , the metagene signature used in that study has a significant intersection with the MAF signature and was found resistant to neoadjuvant chemotherapy. To compare the drug response performance of the $D C N$ metagene set with that of the MAF signature, we used the top 50 genes of the MAF signature in terms of their association with COL11A1 from various cancers (the top genes shown in Table 4) in the same data set. Shown in Additional file 6 and Additional file 7 are two clustering heat maps of expression profiles, one with the MAF signature genes and one with the $D C N$ metagene set, respectively. High expression of the MAF signature genes correlates with lack of response to therapy (identifying 14 samples out of which 12 have lack of response) more than high expression of the $D C N$ metagene set (identifying 12 samples out of which 10 have lack of response), suggesting that the presence of the core genes of the MAF signature provide at least as good indicator of resistance to neoadjuvant chemotherapy. The reason for the drug resistance may simply be that the invasiveness sensed by the MAF signature does not allow the size or extent of the cancer to be reduced prior to surgery.
Table 4 Aggregate list of top genes associated with COL11A1

\begin{tabular}{|c|c|c|c|c|c|}
\hline Probe Set $^{a}$ & Gene & MI & & & \\
\hline 37892_at & COL11A1 & 0.727 & 218469_at & GREM1 & 0.231 \\
\hline 204320_at & COL11A1 & 0.640 & 201261_x_at & $B G N$ & 0.228 \\
\hline 203083_at & THBS2 & 0.418 & 213125_at & $O L F M L 2 B$ & 0.228 \\
\hline 217428_s_at & COL10A1 & 0.386 & 201744_s_at & LUM & 0.228 \\
\hline 205941_s_at & COLIOA1 & 0.373 & 202998_s_at & ENTPD4 & 0.223 \\
\hline 221729_at & COL5A2 & 0.370 & 201438_at & COL6A3 & 0.223 \\
\hline 210511_s_at & INHBA & 0.368 & 212344_at & SULF1 & 0.222 \\
\hline 221730_at & COL5A2 & 0.367 & 209596_at & MXRA5 & 0.221 \\
\hline 213909_at & LRRC15 & 0.342 & 213764_s_at & MFAP5 & 0.221 \\
\hline 212488_at & COL5A1 & 0.337 & 204589_at & NUAK1 & 0.216 \\
\hline 204619_s_at & VCAN & 0.326 & 217762_s_at & RAB31 & 0.216 \\
\hline 209955_s_at & FAP & 0.323 & 213905_x_at & $B G N$ & 0.214 \\
\hline 202311_s_at & COLIA1 & 0.322 & 201150_s_at & TIMP3 & 0.214 \\
\hline 221731_x_at & VCAN & 0.320 & 221541_at & CRISPLD2 & 0.214 \\
\hline 203878_s_at & MMP11 & 0.319 & 217763_s_at & $R A B 31$ & 0.212 \\
\hline 212489_at & COL5A1 & 0.318 & 217430_x_at & COLIA1 & 0.212 \\
\hline 210809_s_at & POSTN & 0.315 & 205422_s_at & ITGBL1 & 0.210 \\
\hline 202310_s_at & COLIA1 & 0.314 & 201147_s_at & TIMP3 & 0.209 \\
\hline 204620_s_at & VCAN & 0.312 & 218468_s_at & GREM1 & 0.209 \\
\hline 202404_s_at & COL1A2 & 0.304 & 217764_s_at & RAB31 & 0.208 \\
\hline 202952_s_at & ADAM12 & 0.300 & 213765_at & MFAP5 & 0.206 \\
\hline 213790_at & ADAM12 & 0.297 & 211668_s_at & PLAU & 0.203 \\
\hline 203325_s_at & COL5A1 & 0.296 & 207173_x_at & $\mathrm{CDH} 11$ & 0.202 \\
\hline 215076_s_at & COL3A1 & 0.295 & 213338_at & TMEM158 & 0.201 \\
\hline 215446_s_at & LOX & 0.293 & 209758_s_at & MFAP5 & 0.199 \\
\hline 210495_x_at & FN1 & & 202363_at & SPOCK1 & 195 \\
\hline 201792_at & AEBP1 & 0.291 & 201148_s_at & TIMP3 & 0.195 \\
\hline 216442_x_at & FN1 & 0.286 & 204051_s_at & SFRP4 & 0.193 \\
\hline 212464_s_at & FN1 & 0.286 & 207172_s_at & $\mathrm{CDH} 11$ & 0.192 \\
\hline 201852_x_at & COL3A1 & 0.286 & 202283_at & SERPINF1 & 0.191 \\
\hline 212353_at & SULF1 & 0.285 & 209335_at & $D C N$ & 0.189 \\
\hline 211719_x_at & FN1 & 0.285 & 204298_s_at & LOX & 0.189 \\
\hline 211161_s_at & COL3A1 & 0.283 & 219655_at & C7orfio & 0.189 \\
\hline 202403_s_at & COLIA2 & 0.278 & 219561_at & COPZ2 & 0.189 \\
\hline 202766_s_at & $F B N 1$ & 0.272 & 219773_at & NOX4 & 0.187 \\
\hline 212354_at & SULF1 & 0.266 & 204464_s_at & EDNRA & 0.186 \\
\hline 219087_at & ASPN & 0.260 & 200974_at & ACTA2 & 0.186 \\
\hline 200665_s_at & SPARC & 0.258 & 202273_at & PDGFRB & 0.185 \\
\hline 215646_s_at & VCAN & 0.257 & 61734_at & RCN3 & 0.185 \\
\hline 211571_s_at & VCAN & 0.256 & 213139_at & SNAI2 & 0.183 \\
\hline 202450_s_at & CTSK & 0.254 & 220988_s_at & $A M A C R$ & 0.182 \\
\hline 206026_s_at & TNFAIP6 & 0.253 & 205713_s_at & $C O M P$ & 0.181 \\
\hline 202765_s_at & FBN1 & 0.247 & 201105_at & LGALSI & 0.181 \\
\hline 203876_s_at & MMP11 & 0.240 & 213869_x_at & THY1 & 0.180 \\
\hline 212667_at & SPARC & 0.239 & 202465_at & PCOLCE & 0.174 \\
\hline 222020_s_at & HNT & 0.239 & 208851_s_at & THY1 & 0.174 \\
\hline 206439_at & EPYC & 0.235 & 209156_s_at & COL6A2 & 0.173 \\
\hline 201069_at & $M M P 2$ & 0.234 & 221447_s_at & GLT8D2 & 0.172 \\
\hline 205479_s_at & PLAU & 0.234 & 204114_at & NID2 & 0.171 \\
\hline 206025_s_at & TNFAIP6 & 0.232 & 205991_s_at & PRRX1 & 0.171 \\
\hline
\end{tabular}

${ }^{\mathrm{a}}$ Affymetrix probe sets. 


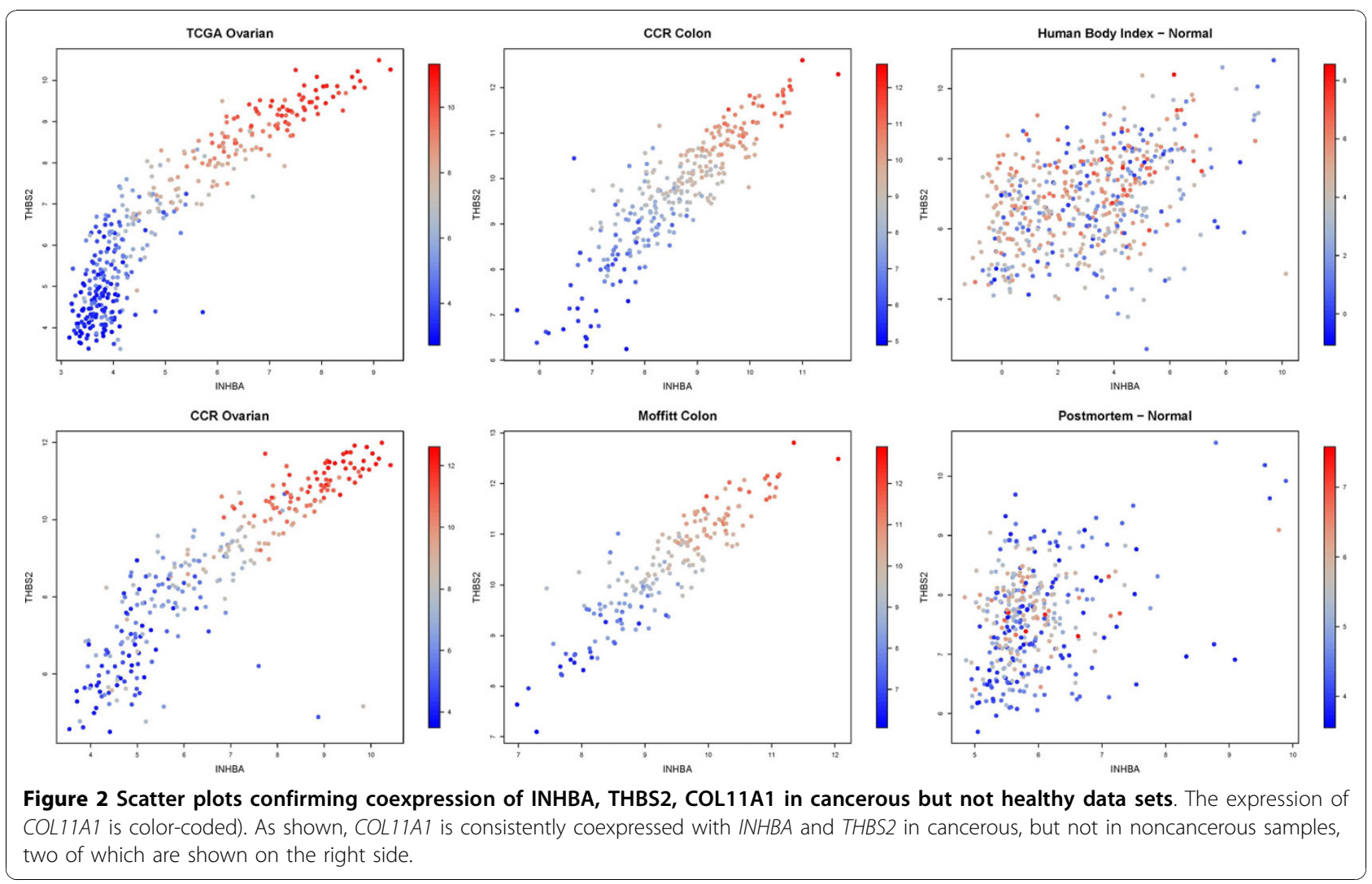

\section{Discussion}

A direct clinical application of these findings is the development of a high-specificity invasion-sensing biomarker product detecting coordinated overexpression of a few top-ranked genes, such as COL11A1, INHBA, and $T H B S 2$, as shown in the scatter plots of Figure 2. A positive result in seemingly low-stage primary tumors will indicate that the disease has obtained the stromal signature and thus has already reached an invasive stage. As described above, the same product can also be used to predict resistance to neoadjuvant chemotherapy.

Of course, the most significant clinical application would be to develop metastasis-inhibiting therapeutics using targets deduced from the biological knowledge provided by the MAF signature. Our top ranked genes strongly suggest that they are produced by myofibroblasts
A

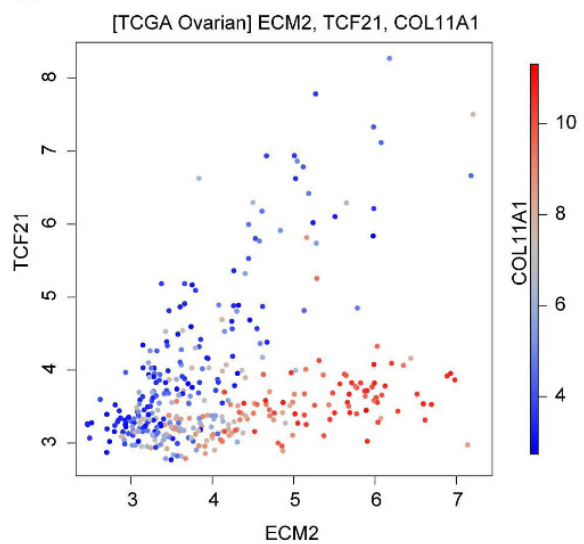

B

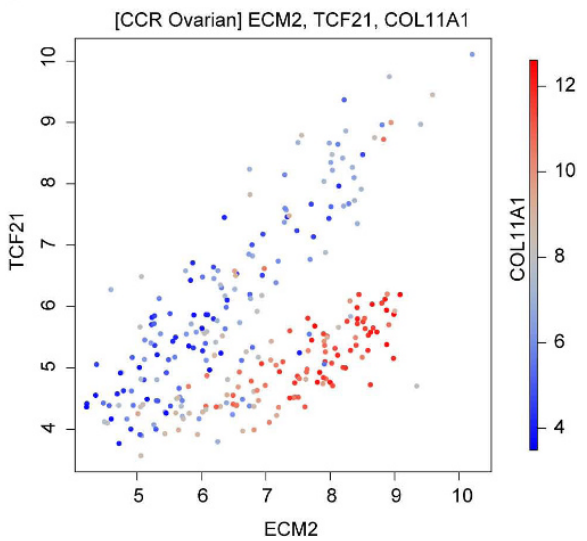

Figure 3 Example of a synergistic pair of genes in two ovarian cancer data sets. 
Table 5 Top-ranked miRNAs in MAF signature in the TCGA ovarian cancer data set

\begin{tabular}{lll}
\hline miRNA & MI & $\begin{array}{l}\text { Up/Down } \\
\text { Regulated }\end{array}$ \\
\hline hsa-miR-22 & 0.204 Up \\
hsa-miR-514-1|hsa-miR-514-2|hsa-miR-514-3 & 0.193 Down \\
hsa-miR-152 & 0.187 Up \\
hsa-miR-508 & 0.168 Down \\
hsa-miR-509-1|hsa-miR-509-2|hsa-miR-509-3 & 0.164 Down \\
hsa-miR-507 & 0.152 Down \\
hsa-miR-509-1|hsa-miR-509-2 & 0.147 Down \\
hsa-miR-506 & 0.146 Down \\
hsa-miR-509-3 & 0.144 Down \\
hsa-miR-214 & 0.128 Up \\
hsa-miR-510 & 0.116 Down \\
hsa-miR-199a-1|hsa-miR-199a-2 & 0.115 Up \\
hsa-miR-21 & 0.112 Up \\
hsa-miR-513c & 0.108 Down \\
hsa-miR-199b & 0.103 Up \\
\hline AlminAs & in
\end{tabular}

All miRNAs have multiple test corrected $p<10^{-16}$ in terms of their association with COL11A1.

or myofibroblast-like cells activated by activin A-induced TGF- $\beta$ signaling and leading to some form of altered proteolysis [21], which results in extracellular matrix remodeling. Supporting this hypothesis are the facts that both INHBA and THBS 2 are involved in TGF- $\beta$ signaling: Activin A (INHBA homodimer) is a TGF- $\beta$ superfamily member (ligand) and THBS2 inhibits activation of TGF-

Table 6 Top-ranked methylation sites in MAF signature in the TCGA ovarian cancer data set

\begin{tabular}{ccc}
\hline Methylation site & MI & Hyper-/Hypomethylated \\
\hline PRAME & 0.223 & Hyper \\
SNAl1 & 0.183 & Hyper \\
KRT7 & 0.158 & Hyper \\
RASSF5 & 0.157 & Hyper \\
FL14816 & 0.155 & Hyper \\
PPL & 0.155 & Hyper \\
CXCR6 & 0.153 & Hypo \\
SLC12A8 & 0.148 & Hyper \\
NFATC2 & 0.148 & Hyper \\
HOM-TES-103 & 0.147 & Hypo \\
ZNF556 & 0.147 & Hyper \\
OCIAD2 & 0.146 & Hyper \\
APS & 0.142 & Hyper \\
MGC9712 & 0.139 & Hyper \\
SLC1A2 & 0.136 & Hyper \\
HAK & 0.131 & Hypo \\
C3Orf18 & 0.13 & Hyper \\
GMPR & 0.13 & Hyper \\
CORO6 & 0.128 & Hyper
\end{tabular}

All methylated genes have multiple-test corrected $p<10^{-16}$ in terms of their association with $\mathrm{COL11A1.}$ $\beta$ by THBS1, which is also present in the MAF signature. Remarkably, activin A is already known to facilitate fibroblast-mediated collagen gel contraction [22]. The role of gene $L R R C 15$ (aka $L I B$ ) appears important but unclear, though it has already been recognized as promoting migration through the extracellular matrix [23]. Versican (VCAN) is an extracellular matrix proteoglycan already known to play a role in metastasis, while MMP11 is one of several matrix metalloproteinases involved in the breakdown of extracellular matrix.

Although each of the MAF signature molecules could serve as a potential therapeutic target, the hypothesis that activin A signaling is at the heart of the MAF mechanism immediately suggests that follistatin (activinbinding protein) could serve as a metastasis inhibitor, which is exactly what recent research [24] indicates. Specifically, lung cancer cell lines transfected with follistatin and injected into immunodeficient mice markedly inhibited metastasis compared with non-transfected cell lines, but the authors of the study recognize that the role of follistatin in cancer metastasis is totally unknown [25]. Our work provides an explanation and suggests that the same could be true for other cancers as well. Further support is provided by the fact that follistatin virtually abolished the fibroblast-mediated collagen gel contraction mentioned earlier [22].

There are several reasons that the core MAF signature has not yet been discovered as a multi-cancer metastasis-associated signature. First, it is essential to identify the correct phenotypic staging threshold recognizing that the signature only exists in a subset of tumors that exceed that particular stage. Indeed, if the threshold in breast cancer was put between stage I and stage II, or between stage II and stage III, rather than between in situ and stage I, the signature would not be apparent. Second, each cancer type may have its own additional features accompanying the MAF signature. For example, in ovarian cancer it is accompanied by sharp downregulation of genes COLEC11, PEG3 and TSPAN8, which is not the case in other cancers. Indeed, the main contribution of our work is the identification of the common multi-cancer "core" signature, from which a universal metastasis-associated biological mechanism can be identified. Third, the MAF signature may be reversible, perhaps as a result of the disappearance of many of the stromal cells in the mature desmoplastic stroma when it is replaced by "acellular" matrix [6]. The presence of the signature in high-stage samples may even paradoxically be associated with longer survival if its reversal is required for further distant metastases (see below).

An important topic of further research is the determination of the precise biological event of interaction of cancer cells with the microenvironment that gives 
rise to the stromal MAF signature and associated invasiveness. Because of the recognized similarities with the mechanisms of wound healing [26], it is likely that this event uses existing wound healing response pathways. For example, it appears to occur very early in breast cancer, late in ovarian cancer, and never in glioblastoma (which is reasonable, because glioblastoma metastasizes extremely rarely). The late appearance of the MAF signature in ovarian cancer and its presence in omental metastases can be explained by the fact that ovarian cancer initially progresses by disseminating locally across mesothelial surfaces and that, contrary to hematogenously metastasizing tumors, initial metastasis is probably carried by the physiological movement of peritoneal fluid to the peritoneum and omentum [27].

Several of the top-ranked genes in the MAF signature (such as thrombospondins, decorin, INHBA itself) are known to be potent anti-angiogenesis mediators. The reversal of the MAF signature would thus facilitate further metastatic dissemination to distant sites. In other words, (a) the desmoplastic MAF signature and (b) angiogenesis, are two independent biological events. The former appears to be based on activin A signaling, as several of the MAF proteins in addition to INHBA are also known inhibitors of the standard TGF- $\beta$ ligand. The reversal of the MAF signature would allow the standard ligand to take over in TGF- $\beta$ signaling, and may thus facilitate further metastasis. These observations provide explanations for the seemingly contradictory observed roles of TGF- $\beta$ signaling inhibiting early cancer but facilitating metastasis.

The possible reversibility of the MAF signature leads to the intriguing hypothesis that perhaps all metastases have, at some point temporarily been there, which explains why we only observe it in a subset of them. This would be particularly exciting, because in that case any metastasis-inhibiting therapeutic intervention targeting the MAF mechanism would be widely applicable to low-stage tumors.

\section{Conclusions}

In conclusion, we have shown that, using purely computational analysis of publicly available biological information, systems biology has revealed the core of a multi-cancer metastasis-associated gene expression signature. In the near future, a vast amount of additional information will become available, including next generation sequencing, miRNA and methylation information for many cancers, which will allow additional computational research building on this work and clarifying the details of the underlying invasion-associated complex biological process.

\section{Additional material}

Additional file 1: Heat map of TCGA ovarian cancer data set. This file contains the result of hierarchical clustering for the TCGA ovarian cancer data set using a particular gene set (see text).

Additional file 2: Implementation of the EVA algorithm. This file contains the description of a low-complexity implementation of the EVA algorithm.

Additional file 3: List of genes resulting from EVA algorithm. This file contains the commonly significant probe sets in four ovarian and colorectal cancers data sets.

Additional file 4: Listing of genes in $\mathbf{9}$ cancer data sets. This file contains a color-coded list of genes in various cancers ranked in terms of the mutual information with COL11A1.

Additional file 5: Heat map of neuroblastoma data set. This file contains the result of hierarchical clustering for the neuroblastoma data set (GSE3960) using the MAF signature genes.

Additional file 6: Heat map of breast cancer data set using MAF signature genes. This file contains the result of hierarchical clustering for the breast cancer data set (GSE4779) using the MAF signature genes.

Additional file 7: Heat map of breast cancer data set using DCN metagene set. This file contains the result of hierarchical clustering for the breast cancer data set (GSE4779) using the DCN metagene set.

\section{Acknowledgements}

Appreciation is expressed to Prof. Jessica Kandel, MD for helpful discussions. This work was supported by university inventor's (DA) research allocation of royalty proceeds from intellectual property unrelated to this paper.

\section{Author details}

${ }^{1}$ Center for Computational Biology and Bioinformatics and Department of Electrical Engineering, Columbia University, New York, NY, USA. ${ }^{2}$ Philips Research North America, Briarcliff Manor, NY, USA.

\section{Authors' contributions}

DA and W conceived of and supervised the study. HK and JW carried out the experiments. DA drafted the manuscript. All authors participated in the study, read and approved the final manuscript.

\section{Competing interests}

The authors declare that they have no competing interests.

Received: 14 June 2010 Accepted: 3 November 2010

Published: 3 November 2010

\section{References}

1. Kalluri R, Weinberg RA: The basics of epithelial-mesenchymal transition. J Clin Invest 2009, 119:1420-1428.

2. Thiery JP: Epithelial-mesenchymal transitions in tumour progression. Nat Rev Cancer 2002, 2:442-454.

3. Albini A, Sporn MB: The tumour microenvironment as a target for chemoprevention. Nat Rev Cancer 2007, 7:139-147.

4. Stover DG, Bierie B, Moses HL: A delicate balance: TGF-beta and the tumor microenvironment. J Cell Biochem 2007, 101:851-861.

5. Kalluri R, Zeisberg M: Fibroblasts in cancer. Nat Rev Cancer 2006, 6:392-401.

6. Weinberg RA: The biology of cancer New York: Garland Science; 2007.

7. Bignotti E, Tassi RA, Calza S, Ravaggi A, Bandiera E, Rossi E, Donzelli C, Pasinetti B, Pecorelli S, Santin AD: Gene expression profile of ovarian serous papillary carcinomas: identification of metastasis-associated genes. Am J Obstet Gynecol 2007, 196:245, e241-211.

8. Tibshirani $R$, Walther $G$, Hastie $T$ : Estimating the number of clusters in a dataset via the Gap statistic. J R Statist SoC B 2001, 63:411-423.

9. Anastassiou D: Computational analysis of the synergy among multiple interacting genes. Mol Syst Biol 2007, 3:83. 
10. Tothill RW, Tinker AV, George J, Brown R, Fox SB, Lade S, Johnson DS, Trivett MK, Etemadmoghadam D, Locandro B, et al: Novel molecular subtypes of serous and endometrioid ovarian cancer linked to clinical outcome. Clin Cancer Res 2008, 14:5198-5208.

11. Jorissen RN, Gibbs P, Christie M, Prakash S, Lipton L, Desai J, Kerr D, Aaltonen LA, Arango D, Kruhoffer M, et al: Metastasis-Associated Gene Expression Changes Predict Poor Outcomes in Patients with Dukes Stage B and C Colorectal Cancer. Clin Cancer Res 2009, 15:7642-7651.

12. Smith JJ, Deane NG, Wu F, Merchant NB, Zhang B, Jiang A, Lu P, Johnson JC, Schmidt C, Bailey CE, et al: Experimentally derived metastasis gene expression profile predicts recurrence and death in patients with colon cancer. Gastroenterology 2010, 138:958-968.

13. Schuetz CS, Bonin M, Clare SE, Nieselt K, Sotlar K, Walter M, Fehm T, Solomayer E, Riess O, Wallwiener D, et al: Progression-specific genes identified by expression profiling of matched ductal carcinomas in situ and invasive breast tumors, combining laser capture microdissection and oligonucleotide microarray analysis. Cancer Res 2006, 66:5278-5286.

14. Vecchi M, Nuciforo P, Romagnoli S, Confalonieri S, Pellegrini C, Serio G, Quarto M, Capra M, Roviaro GC, Contessini Avesani E, et al: Gene expression analysis of early and advanced gastric cancers. Oncogene 2007, 26:4284-4294.

15. Badea L, Herlea V, Dima SO, Dumitrascu T, Popescu I: Combined gene expression analysis of whole-tissue and microdissected pancreatic ductal adenocarcinoma identifies genes specifically overexpressed in tumor epithelia. Hepatogastroenterology 2008, 55:2016-2027.

16. Apte MV, Wilson JS: The Desmoplastic Reaction in Pancreatic Cancer: An Increasingly Recognised Foe. Pancreatology 2007, 7:378-379.

17. Chong IW, Chang MY, Chang HC, Yu YP, Sheu CC, Tsai JR, Hung JY, Chou SH, Tsai MS, Hwang JJ, Lin SR: Great potential of a panel of multiple hMTH1, SPD, ITGA11 and COL11A1 markers for diagnosis of patients with non-small cell lung cancer. Oncol Rep 2006, 16:981-988.

18. Schmalbach CE, Chepeha DB, Giordano TJ, Rubin MA, Teknos TN, Bradford CR, Wolf GT, Kuick R, Misek DE, Trask DK, Hanash S: Molecular profiling and the identification of genes associated with metastatic oral cavity/pharynx squamous cell carcinoma. Arch Otolaryngol Head Neck Surg 2004, 130:295-302

19. Watkinson J, Liang KC, Wang X, Zheng T, Anastassiou D: Inference of regulatory gene interactions from expression data using three-way mutual information. Ann N Y Acad Sci 2009, 1158:302-313.

20. Farmer $P$, Bonnefoi $H$, Anderle $P$, Cameron D, Wirapati $P$, Becette $V$, Andre S, Piccart M, Campone M, Brain E, et al: A stroma-related gene signature predicts resistance to neoadjuvant chemotherapy in breast cancer. Nat Med 2009, 15:68-74.

21. Kessenbrock K, Plaks V, Werb Z: Matrix metalloproteinases: Regulators of the tumor microenvironment. Cell 2010, 141:52-67.

22. Ohga E, Matsuse T, Teramoto S, Ouchi Y: Activin receptors are expressed on human lung fibroblast and activin A facilitates fibroblast-mediated collagen gel contraction. Life Sci 2000, 66:1603-1613.

23. Satoh K, Hata M, Shimizu T, Yokota H, Akatsu H, Yamamoto T, Kosaka K, Yamada T: Lib, transcriptionally induced in senile plaque-associated astrocytes, promotes glial migration through extracellular matrix. Biochem Biophys Res Commun 2005, 335:631-636.

24. Talmadge JE: Follistatin as an inhibitor of experimental metastasis. Clin Cancer Res 2008, 14:624-626.

25. Ogino H, Yano S, Kakiuchi S, Muguruma H, Ikuta K, Hanibuchi M, Uehara H, Tsuchida K, Sugino $H$, Sone S: Follistatin suppresses the production of experimental multiple-organ metastasis by small cell lung cancer cells in natural killer cell-depleted SCID mice. Clin Cancer Res 2008, 14:660-667.

26. Schafer M, Werner S: Cancer as an overhealing wound: an old hypothesis revisited. Nat Rev Mol Cell Biol 2008, 9:628-638.

27. Lengyel E: Ovarian cancer development and metastasis. Am J Pathol 2010, 177:1053-1064

\section{Pre-publication history}

The pre-publication history for this paper can be accessed here: http://www.biomedcentral.com/1755-8794/3/51/prepub

doi:10.1186/1755-8794-3-51

Cite this article as: Kim et al:: Multi-cancer computational analysis reveals invasion-associated variant of desmoplastic reaction involving INHBA, THBS2 and COL11A1. BMC Medical Genomics 2010 3:51.

\section{Submit your next manuscript to BioMed Central and take full advantage of:}

- Convenient online submission

- Thorough peer review

- No space constraints or color figure charges

- Immediate publication on acceptance

- Inclusion in PubMed, CAS, Scopus and Google Scholar

- Research which is freely available for redistribution 